SCIREA Journal of Clinical Medicine

ISSN: 2706-8870

http://www.scirea.org/journal/CM

January 10, 2022

Volume 7, Issue 1, February 2022

https://doi.org/10.54647/cm32748

\title{
SECONDARY HEMOPHAGOCYTIC \\ LYMPHOHISTIOCYTIC SYNDROME IN SEVERE COVID-19 INFECTION: DIAGNOSTIC CHALLENGES \\ AND A CASE DISCUSSION
}

\author{
SAMIA HASSAN RIZK \\ PROFESSOR OF CLINICAL PATHOLOGY, FACULTY OF MEDICINE, CAIRO \\ UNIVERSITY \\ Correspondence: SAMIA HASSAN RIZK \\ Address: 25 ABDEL HALIM HUSSEIN ST., 21311, ELMOHANDESEEN, GUIZA, EGYPT \\ Email:rizksh@gmail.com
}

\section{SUMMARY}

The hemophagocytic lymphohistiocytic syndrome (HLH) has been reported in critically ill patients with confirmed sars-cov-2 infection and contributes to their morbidity and mortality. Precise criteria for diagnosis and monitoring of $\mathrm{HLH}$ in these patients are essential for improving their clinical outcomes. The diagnosis of HLH depends on several clinical, laboratory, and morphologic parameters according to the HLH-2004 guidelines and its update for adult patients. Recently, the H-score tool provides a quantitative score that can be used to monitor clinical severity and detect early cases with incomplete criteria. Other differential diagnoses which should be considered in COVID-19 infection associated with 2ry HLH are sepsis and septic shock, and cytokine release syndrome (CRS) due to a wide overlap of 


\section{SECONDARY HLH IN COVID-19 INFECTION}

clinicopathologic findings. The pathogenesis of COVID-19 associated 2ry HLH and whether a direct causal relationship exists between them are areas of intense investigation. A case of 65 years male with a documented COVID-19 disease and an associated 2ry HLH, 1 month following infection is reported, using both the HLH-2004 guidelines and a high H-score $(>169)$. We recommend the use of a wide laboratory diagnostic panel for precise confirmation of HLH in all patients with early signs of clinical deterioration or underlying morbidity. These patients should be regularly monitored according to their rate of decline to allow for early intervention.

Keywords: COVID-19, HEMOPHAGOCYTOSIS, HLH, BONE MARROW MACROPHAGE, H-SCORE, PATHOGENESIS

\section{INTRODUCTION}

SARS-CoV-2 is a novel human pathogen that may interact with the host antiviral defense uniquely.

About $10-15 \%$ of patients with covid-19 progress to severe disease and about $5 \%$ become critically ill. People recovering from acute illness can have lasting symptoms for weeks or even months, though they are not infectious this state has been termed post-covid condition. But, no consensus about this definition is still widely accepted. These cases may develop medical complications with lasting health effects. ${ }^{1}$ Furthermore, the post-acute sequelae of covid-19 represent an emerging public health challenge with a great global burden.

The underlying pathogenesis of these post-acute sequelae is related to a combination of the initial sars-cov-2 viral insult together with the ongoing abnormalities in the host immunoregulatory systems that can affect almost all body organs.

Hemophagocytic lymphohistiocytic syndrome (HLH) has been reported in critically ill patients with confirmed sars-cov-2 infection in some studies which was suggested as a potential trigger. ${ }^{2}$

Precise diagnostic criteria and monitoring for HLH in these critically ill patients are important for improving their clinical outcomes. We report the presenting clinical and laboratory features in a patient with severe COVID-19 disease, 1 month following his 


\section{SECONDARY HLH IN COVID-19 INFECTION}

documented infection. The diagnostic approach, clinicopathologic findings, and possible differential diagnoses are discussed.

\section{CASE DESCRIPTION}

A 65 years old male was diagnosed with a documented COVID-19 infection one month before his current presentation and hospital administration. He didn't recover and had progressive clinical deterioration. The patient received COVID 19 VACCINE 2 months before presentation. His past medical history was unremarkable.

He presented to the hospital one month following his positive viral test with:

Dehydration, hypotension, AKI on top of CKD, anorexia lasting for 2 weeks. He had metabolic acidosis (PH 7.2, HCO3 10, PCO2 23), increased uric acid, RBS 256. Ultrasound examination showed coarse mild hepatomegaly, marked splenomegaly, and grade I nephropathy. He received several treatments including antibiotics, NaHCO3, EPO4000, G$\mathrm{CSF}$, and platelet transfusion.

\section{LABORATORY FINDINGS:}

- $\quad$ Tests for other common viruses were negative: HCV AB, HBS AG, HIV

- $\quad$ Complete blood count: HB 8.3, RBCS 3.2, HCT 24.9, MCV 77.8, MCH 25.9, MCHC 33.3, RDW 19.6, PLAT 29, WBCS: 2.2. DIFF: BASO 0, EOS 0, STAFF 4, SEG 71, LYMPH 10, MONO 15

- $\quad$ Blood Chemistry:

- $\quad$ FERRITIN $>450$

- $\quad$ BILIRUBIN 3.7, DIRECT2.3, INDIRECT 1.4

- $\quad$ ALT 92, AST 86

- TOtal PROTEIN 4.2, ALBUMin 2.3

- $\quad$ UREA 284, BUN 133, CREAT7.3

- $\quad$ URIC ACID 10.8

- $\quad$ CA TOT 7, CA IO 3.6 LOW

- $\quad \mathrm{NA} 131, \mathrm{~K} 4.8,6.4, \mathrm{MG} 2.2$

- LDH373 H

- CRP 166H

- TRIGLYCERIDES $375 \mathrm{H}$ 


\section{SECONDARY HLH IN COVID-19 INFECTION}

- $\quad$ D-DIMER $1.5+$

- BONE MARROW EXAMINATION WAS ORDERED BECAUSE OF HIS PANCYTOPENIA AND SHOWED: An overall mildly hypocellular marrow for age, with normal megakaryocytes moderate reduction of Erythroid and myeloid elements with few scattered small erythroid foci and differentiated myeloid elements that showed a mild shift to left. Few interstitially scattered lymphocytes \& plasma cells are seen with occasional Russel bodies. Many interstitially scattered macrophages are encountered with hemophagocytosis. Bone marrow IHC showed: CD 68: prominent increase of scattered positive cells. CD3: many interstitially scattered CD3 positive cells and interstitially scattered CD20 positive cells with some aggregates. Other markers were non-remarkable including CD38: few interstitially scattered positive cells, CD138 positive cells constituted overall about 5-8\%. Both CD56 and CD30 were negative. Only a few interstitially scattered CD10 positive cells were seen.

In summary, The IHC pattern reveals a prominent increase of macrophage elements (CD 68 positive) \& a prominent increase of mixed B\& $T$ together with a mild increase of plasma cells with no aberrant expression.

Images of bone marrow morphology and IHC are demonstrated in figure-1.

Figure 1: The bone marrow histopathology and IHC of the studied case.

A. BM, HE SHOWING HYPOCELLULARITY (X5)

B. BM, HE SHOWING PROMINENT MACROPHAGE PROLIFERATION (X10)

C. BM, HE SHOWING PROMINENT MACROPHAGE PROLIFERATION WITH HEMOPHAGOCYTOSIS OF A LYMPHOCYTE (POINTED TO BY THE ARROW) (X10)

D. BM, CD68 IHC SHOWING MANY SCATTERED POSITIVE CELLS (x10)

E. BM, CD20 IHC SHOWING MANY POSITIVE CELLS AND A SINGLE AGGREGATE (x10)

F. BM, CD3 IHC SHOWING MANY SCATTERED POSITIVE CELLS (x10)

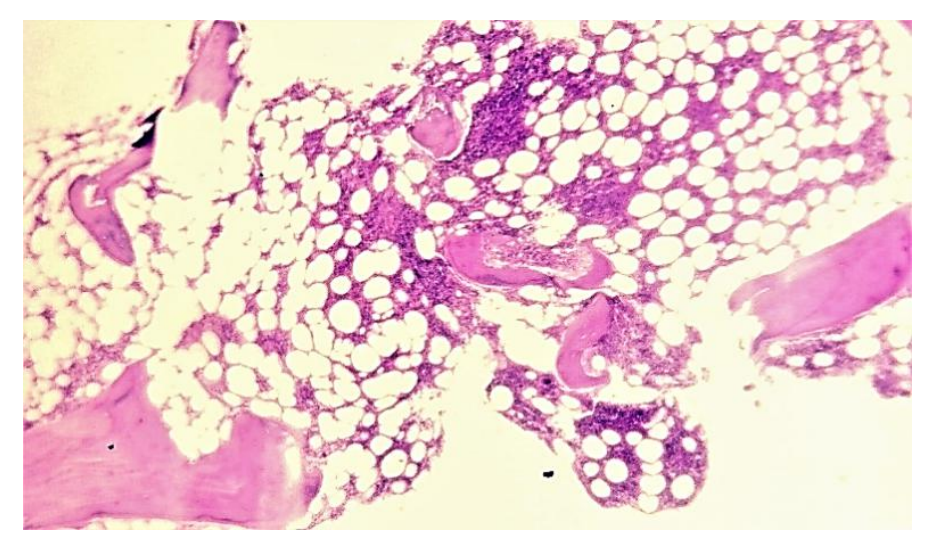

Figure 1-A 


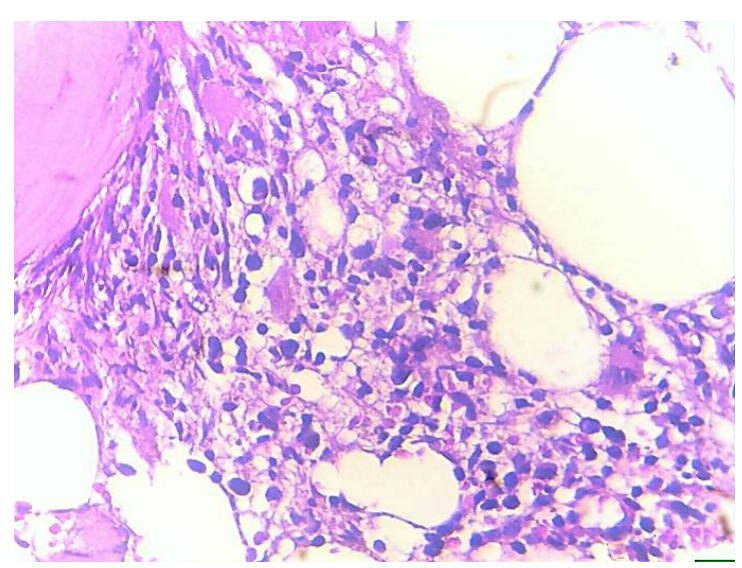

Figure 1-B

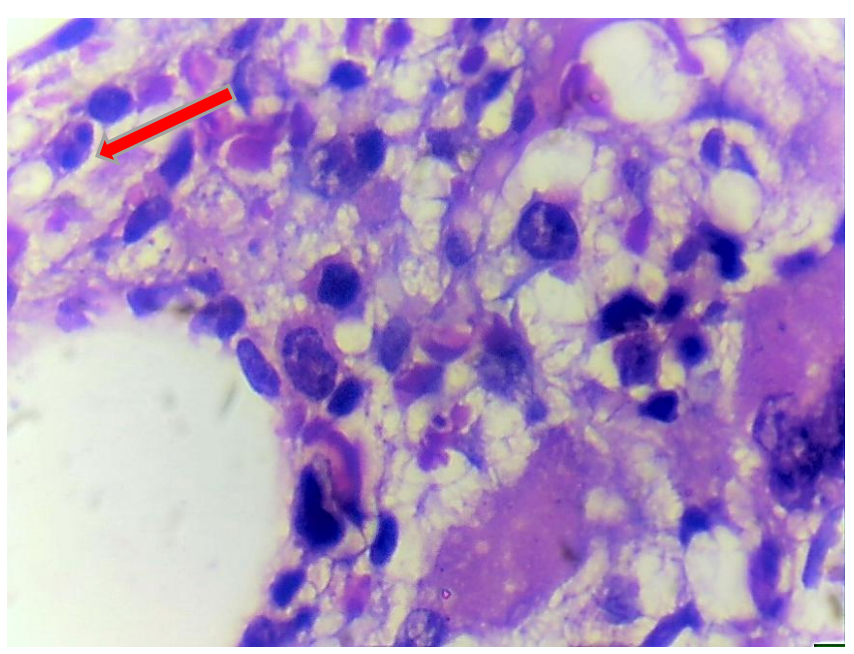

Figure 1-C

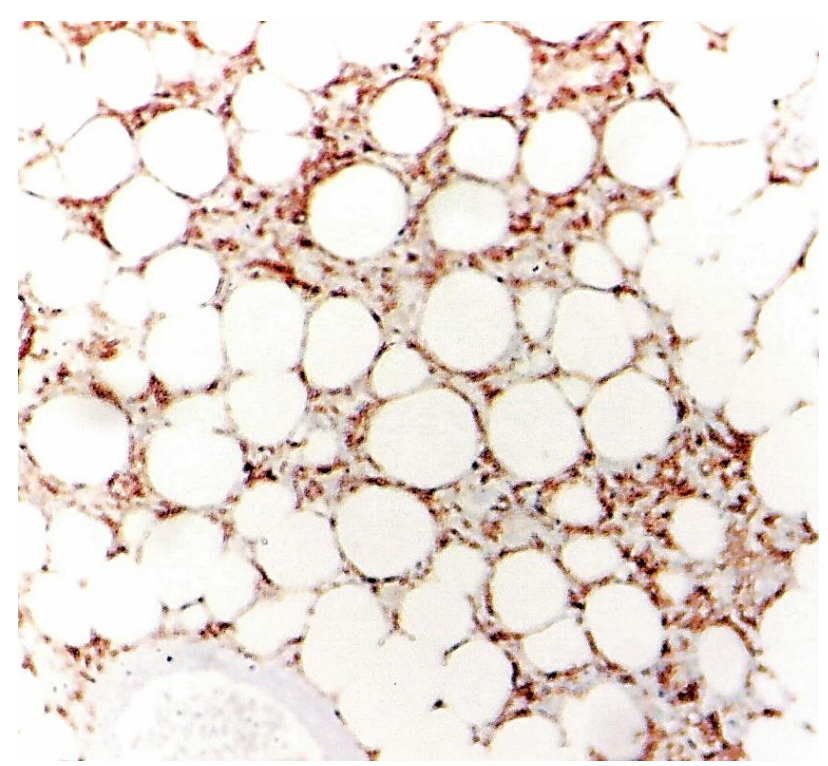

Figure 1-D 


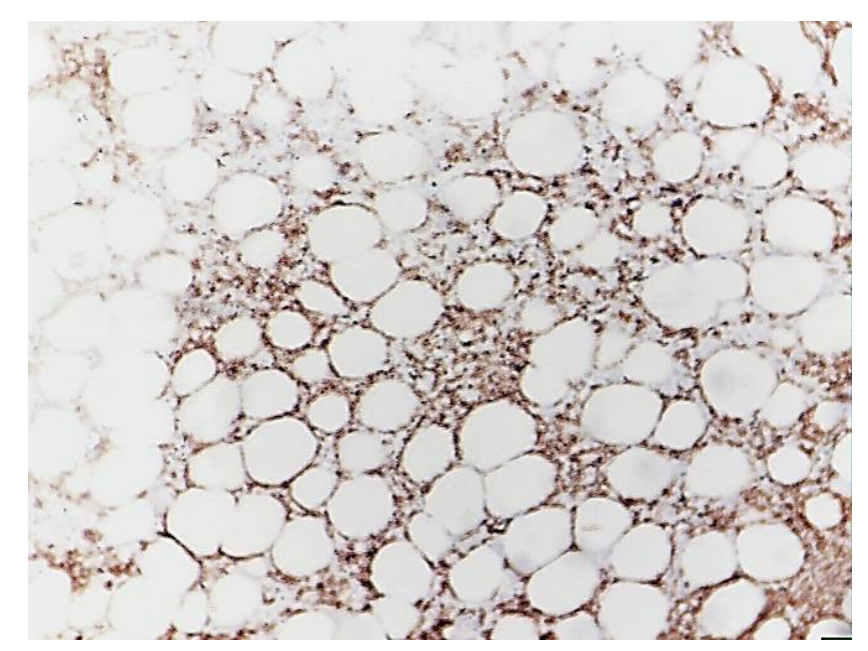

Figure 1-E

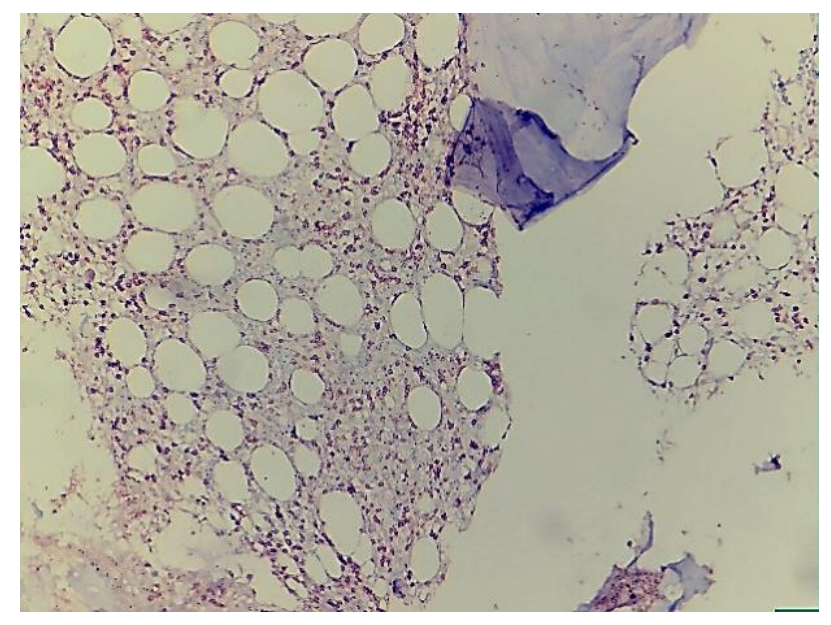

Figure 1-F

The H SCORE was calculated to be 248 (CUT-OFF: 169) with a PROBABILITY OF 99.3 of HAVING HLH

\section{DISCUSSION}

Hemophagocytic lymphohistiocytosis (HLH) is a severe life-threatening inflammatory condition associated with intense cytokine release (cytokine storm) seen also in severe covid19 infection and the clinical characteristics of both may overlap. Clinical management must differentiate both conditions.

The diagnosis of HLH depends on many clinical, laboratory, and morphologic parameters to fulfill the criteria of the HLH-2004 guidelines and its update for adult patients. ${ }^{3}$ The recently 


\section{SECONDARY HLH IN COVID-19 INFECTION}

developed H-score tool is a probability estimate of HLH occurrence in a particular patient. It is based on the weighted measurement of some clinical and laboratory findings including fever, hepatosplenomegaly, cytopenias, ferritin, triglyceride, liver enzymes, etc., and presence of hemophagocytosis. ${ }^{4}$

In one series, high fever, hyperferritinemia, hypertriglyceridemia, bi- or pancytopenia, and hypofibrinogenemia were the most distinguishing between HLH-associated and non-HLHassociated covid-19 infection. ${ }^{5}$

our patient presented 1 month following a documented covid-19 infection with a rapid deterioration of the clinical status, fever not responding to antibiotics, and hepatosplenomegaly. He had multiple metabolic and organ dysfunctions including dehydration, hypotension metabolic acidosis, high blood glucose, raised liver enzymes and bilirubin, low serum protein and albumin and poor renal function, electrolyte disturbances. Positive biochemical inflammatory markers included high, ferritin, CRP, LDH, and D-dimer levels. Serum triglycerides were elevated. His blood picture revealed pancytopenia including marked thrombocytopenia.

The presence of organomegaly and positive inflammatory markers, together with the raised LDH, serum triglycerides, and serum ferritin in this patient with severe COVID-19 infection suggested an associated HLH.

His bone marrow examination showed hypocellularity with normal megakaryocytes, moderate reduction of erythroid and myeloid elements with a mild myeloid shift to left and interstitially scattered lymphocytes and plasma cells together with a striking increase of scattered macrophages with hemophagocytosis.

Because of the patient's grave condition, there was a clinical suspicion of a hematologic malignancy especially with the presence of organomegaly and peripheral pancytopenia. Immunohistochemistry was performed and showed mainly a prominent increase of CD 68 positive macrophages with highlighted hemophagocytosis together with an increase of both CD3 positive and CD20 positive lymphocytes, the latter showed few aggregates. CD138 positive plasma cells were mildly increased. Other markers were non-remarkable.

Bone marrow examination is not commonly required in COVID-19 patients. In the few COVID-19 cases reported by Prieto-Pérez the bone marrow revealed comparable changes but was hypercellular. ${ }^{6}$ In addition, there was also myeloid predominance with some degree of a shift to the left. In their series, macrophages showed mainly erythrophagocytosis, 


\section{SECONDARY HLH IN COVID-19 INFECTION}

hemosiderin deposition, and occasional multinucleate forms. No granulomas were noted in any of their cases.

Immunohistochemistry in most samples showed aggregates of CD8+ interstitial lymphocytes and occasionally interstitial lymphoid primary follicles together with plasma cells in the range of 1 to $4 \%$. In their 3 COVID-19 positive patients, all criteria were consistent with the diagnosis of secondary HLH.

The evident increase of both B and T lymphocytes in our patient is not further investigated to explore its pathogenesis and thus currently no explanation could be given. However, the parallel increase of both lymphocyte types didn't generally favor a neoplastic process and a reactive proliferation was the most logical.

The morphologic detection of hemophagocytosis is a diagnostic criterion for HLH. However, it is not specific and can also be seen in patients with sepsis and other inflammatory conditions. Furthermore, not detecting these cells doesn't exclude HLH in presence of a high $\mathrm{H}$-score as may occur in the early stages.

It was suggested that hemophagocytosis of nucleated cells rather than only erythroid elements, and especially multiple cell phagocytosis by a single macrophage, are more specific for HLH. ${ }^{6}$ This was consistent with the findings in our patient.

The findings in our patient were thus consistent with 2ry HLH according to the HLH-2004 diagnostic criteria. ${ }^{3}$ In addition, the calculated H-score was $>169$ further supporting our diagnosis.

Whether the diagnosed HLH is triggered by the COVID-19 infection or is due to some other cause remains questionable.

Even with a positive COVID-19 test, other viral triggers of HLH should be excluded e.g., EBV, CMV, etc. In our patient common viruses were excluded including HBV, HCV, and HIV, which favored the possibility of a COVID-19-associated HLH syndrome.

The other question is what are the possible differential diagnoses in our patients? Of special consideration are severe COVID-19 infection, sepsis and septic shock, and cytokine release syndrome (CRS). ${ }^{7}$

One of the diagnostic challenges is the overlap of many of the clinical characteristics of severe COVID-19 infection with those of HLH. Patients in both conditions may show 


\section{SECONDARY HLH IN COVID-19 INFECTION}

deterioration of liver functions, and rapidly progressing multi-organ failure. In addition, patients with HLH can also develop respiratory symptoms or ARDS. ${ }^{8}$

An overlap of many laboratory findings is also observed including lymphopenia, raised Ddimer levels, increased CRP, high serum ferritin, and LDH levels as well as increased levels of other markers of macrophage activation (e.g., $\beta 2$-microglobulin), cytokines' (e.g., interferon [IFN]- $\gamma, \mathrm{TNF}-\alpha$ ), and IL-6. Coagulopathies can also occur in both conditions including; increased fibrinogen degradation and decreased fibrinogen levels being related to an increase in proinflammatory cytokines. ${ }^{9}$

However, certain features may help to differentiate both conditions i.e., HLH and severe COVID-19 infection particularly, organomegaly, cytopenia, and elevated triglycerides. ${ }^{10}$

A diagnostic challenge is to fulfill the recommended standard criteria based on the revised HLH 2004 guideline and its recent update for adult patients. ${ }^{3}$ This guideline defines 5 main criteria (fever, splenomegaly, cytopenia, hypertriglyceridemia, and/or hypofibrinogenemia, and hemophagocytosis) and 3 additional items namely low/absent NK-cell activity, hyperferritinemia, and high-soluble IL-2-receptor levels. ${ }^{3}$ The diagnosis requires the presence of at least 5 of these eight criteria except when positive family history or HLHspecific molecular abnormality is also detected. The Absence of hemophagocytosis does not exclude a diagnosis.

The recent scoring tool (H-score) was proposed for the diagnosis of HLH and is freely available online. ${ }^{11}$ Its criteria mainly correspond to the primary HLH and may not always fit into the secondary form.

In the future another set of criteria might be needed for the diagnosis of COVID-19associated HLH, if more evidence suggests some unique features. However, with the current evidence HLH should be suspected in patients with worsening or severe COVID-19 and early diagnosis could potentially be made using a panel of diagnostic tests based on the $\mathrm{H}$ score.

Sepsis is another life-threatening organ dysfunction caused by a dysregulated response to infection. Its diagnosis depends on a suspected or documented infection and an acute increase of $\geq 2$ SOFA (Sequential [Sepsis-Related] Organ Failure Assessment Score) points (a proxy for organ dysfunction). However, the current criteria do not allow its clear distinction from HLH. 


\section{SECONDARY HLH IN COVID-19 INFECTION}

The cytokine release syndrome (CRS) can also be triggered by infections and can present with similar symptoms and laboratory abnormalities to HLH and can thus complicate diagnosis. ${ }^{9}$

Therefore, an extended laboratory tests' panel was suggested for a more precise distinction of HLH including: ${ }^{11}$ Throat swab or another respiratory sampling to identify SARS-CoV-2 RNA by PCR; hematology examination (blood count, lymphocyte subpopulation); tests for common respiratory viruses, mycoplasma, chlamydia, and tuberculosis; comprehensive biochemistry panel (liver and renal function tests; myocardial enzyme and myoglobin levels), urine test, CRP, procalcitonin, lactate, D-dimer levels, coagulation profile, measurement of inflammatory factors (IL-6, IL-10, TNF- $\alpha$ ), complement; and anti-acid staining. The assessment of ferritin, fibrinogen, triglycerides, total protein/albumin, and LDH to laboratory tests would allow early identification of patients with a cytokine storm syndrome.

Since the effectiveness of HLH therapy is time-dependent; it should be started as soon as possible. It was proposed that patients with worsening or severe COVID-19 should undergo a diagnostic panel of tests and undergo regular monitoring to enable rapid intervention, with the treatment being modified based on the underlying cause and course of the disease. ${ }^{11}$

A diagnostic scheme for patients with COVID-19 was proposed by Opoka-Winiarska et al and is depicted in figure $2 .{ }^{11}$ 


\section{SECONDARY HLH IN COVID-19 INFECTION}

Figure 2: Proposed diagnostic scheme for patients with COVID-19. ${ }^{11}$

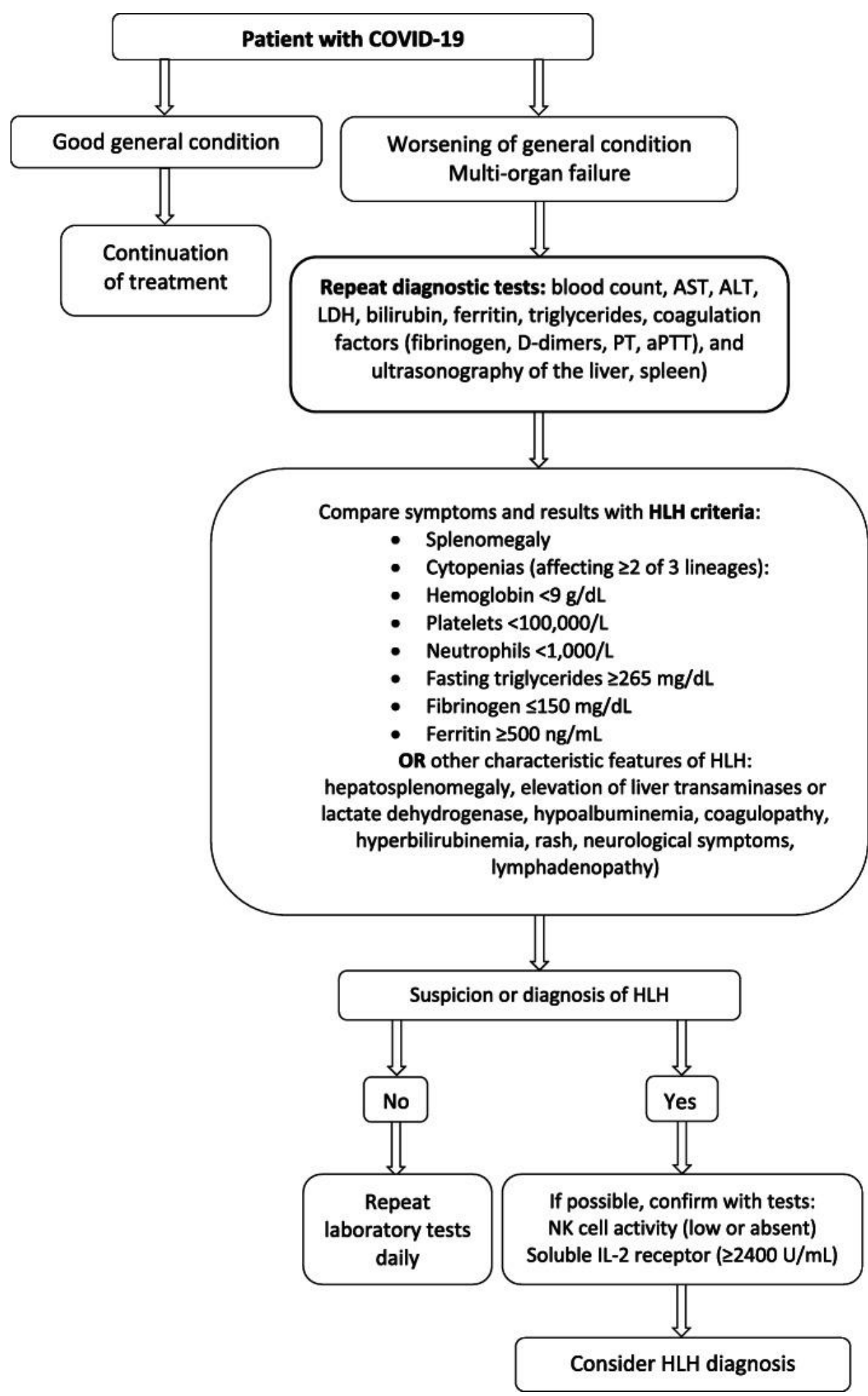

Proposed diagnostic scheme for patients with COVID-19. Abbreviations: ALT, alanine aminotransferase; AST, aspartate aminotransferase; COVID-19, coronavirus disease 2019; HLH, hemophagocytic lymphohistiocytosis; IL-2, interleukin-2; LDH, lactate dehydrogenase; NK, natural killer; PT, prothrombin time; aPPT, activated partial thromboplastin time 


\section{SECONDARY HLH IN COVID-19 INFECTION}

The pathogenesis of HLH in patients with severe COVID-19 infection is still an area of intense investigation. It is known to be associated with severe viral diseases. Other triggering factors include malignancies and a variety of other infections (viral, bacteria, fungi, or parasites). HLH represents the tissue counterpart of the macrophage activation syndrome (MAS) which is characterized by the overactivation of cytotoxic $\mathrm{T}$ cells with hemophagocytosis and a massive release of inflammatory cytokines. It has been classified among the cytokine storm syndromes which are characterized by the overactivation of tissue macrophages with the release of a storm of cytokines that leads to rapidly progressing organ dysfunction. The increased macrophage activation is reflected in the elevation of soluble CD163 levels. ${ }^{11}$

It is hypothesized that COVID-19 infection triggers a systemic immune-inflammatory disease. ${ }^{6}$ However, the role of the COVID-19 virus in triggering HLH is still speculative. In a study by YANG et.al. of 268 critically ill patients with confirmed SARS-CoV-2 infection, $6.3 \%$ of patients were diagnosed as HLH (H-score >169). ${ }^{7}$ The interval from the onset of a symptom of COVID-19 to HLH diagnosis was 19 days and all patients died within 4 days of diagnosis. After excluding other potential triggers in these patients, the authors suggested SARS-CoV-2 as a potential trigger for HLH. However, Cytokine storm syndromes and immunosuppression should be also considered in patients with severe COVID-19 disease. ${ }^{2}$

Currently, not enough evidence supports a causal relation of SARS-CoV-2 to HLH, which is well-conceived as a potential risk factor for mortality in these patients. Physicians caring for critically ill patients with COVID-19 must be aware of the possibility of this complication and pay attention to its important indicators. Molecular analysis of the immune abnormalities in individuals with COVID-19 may be useful in the future in predicting which individuals are at the greatest risk of a cytokine storm and HLH. ${ }^{5}$

\section{CONCLUSION AND RECOMMENDATIONS}

Severe COVID-19 disease may be associated with HLH which increases the gravity and mortality in these patients. Precise diagnosis of HLH in these cases is critical to early intervention and improved outcomes.

The standard HLH 2004 guidelines and its adult update provide a sufficient scheme when manifestations are well-developed. However, it can miss early and atypical presentations. 
The weighted score clinical tool (H-score) provides a quantitative parameter that can be monitored over time. It is freely available online.

Exclusion of other common viruses, hematologic malignancy, and exclusion of similar chronic inflammatory conditions with overlapping features are important.

We recommend the use of a wide panel of diagnostic laboratory tests for a precise confirmation of HLH. This applies to all patients of COVID-19 disease who show early signs of clinical deterioration or have underlying morbidity and should be regularly monitored according to their rate of decline.

\section{REFERENCES}

[1] EPI-WIN: WHO Information Network for Epidemics (2021). THE LATEST ON THE COVID-19 GLOBAL SITUATION \& LONG-TERM SEQUELAE L. LAST UPDATE ON LONG-TERM EFFECTS OF VOVID-19. 26 March 2021. 소스 communication (who. int)

[2] Prilutskiy, A., Kritselis, M., Shevtsov, A., Yambayev M., Vadlamudi, C., et.al. (2020). SARS-CoV-2 Infection-Associated Hemophagocytic Lymphohistiocytosis an Autopsy Series with Clinical and Laboratory Correlation. Am J Clin Pathol. ; XX:0-0 DOI: 10.1093/AJCP/AQAA124

[3] Histiocytosis Society (2004). HLH-2004 (UK No: LCH 2006 02) Hemophagocytic Lymphohistiocytosis Study Group Treatment Protocol of the Second International HLH Study 2004. Histiocyte Society (bspho.be)

[4] Fardet L, Galicier L, Lambotte O, et al. (2014). Development and validation of the Hscore, a score for the diagnosis of reactive hemophagocytic syndrome. Arthritis Rheumatol. 66:2613- 2620. doi:10.1002/art.38690.

[5] Ramakrishnan RK, Kashour T, Hamid Q, Halwani R, and Tleyjeh IM (2021) Unraveling the Mystery Surrounding Post-Acute Sequelae of COVID-19. Front. Immunol. 12:686029. DOI: 10.3389/fimmu.2021.686029. Front. Immunol., 30 June | https://doi.org/10.3389/fimmu.2021.686029

[6] Prieto-Pérez L., Fortes J., Soto C., Vidal-González A., et.al. (2020). Histiocytic hyperplasia with hemophagocytosis and acute alveolar damage in COVID-19 infection. Modern Pathology. 33:2139-2146 https://doi.org/10.1038/s41379-020-0613-1 
[7] YANG K., XING M., JIANG L., et.al. (2021). Infection-associated Hemophagocytic Syndrome in Critically Ill Patients with COVID-19. Current Medical Science DOI https://doi.org/10.1007/s11596-021-2315-4 41(1):39-45

[8] Korompoki, M. Gavriatopoulou, R.S. Hicklen et al. (2021). Epidemiology and organspecific sequelae of post-acute COVID19: A narrative review. Journal of Infection 83 (2021) 1-1. https://doi.org/10.1016/j.jinf.2021.05.004 0163-4453/@ 2021 The British Infection Ass

[9] Ladds E, Rushforth A, Wieringa S, Taylor S, Rayner C, Husain L, et al. Persistent symptoms after COVID-19: a qualitative study of 114 "long COVID" patients and draft quality principles for services. BMC Health Serv Res 2020;20(1). doi:10. 1186/s12913020-06001-y

[10] Debaugnies F, Mahadeb B, Ferster A, et.al. (2016). Performances of the H-score for diagnosis of hemophagocytic lymphohistiocytosis in adult and pediatric patients. Am $\mathrm{J}$ Clin Pathol. 145:862-870. doi:10.1093/ajcp/aqw076.

[11] Opoka-Winiarska, V., Grywalska E., and Roliński J., (2020). Could hemophagocytic lymphohistiocytosis be the core issue of severe COVID-19 cases? BMC Medicine. 18:214 https://doi.org/10.1186/s12916-020-01682-y 